\title{
Only connect
}

\section{An elegant demonstration that the Universe is made of quantum graphs.}

\section{Greg Egan}

ए. M. Forster's famous advice to 'Only connect!' is beginning to look superfluous. A theory in which the building blocks of the Universe are mathematical structures - known as graphs - that do nothing but connect has just passed its first experimental test.

A graph can be drawn as a set of points, called nodes, and a set of lines joining the nodes, called edges. Details such as the length and shape of the edges are not part of the graph, though: the only thing that distinguishes one graph from another is the connections between the nodes. The number of edges that meet at any given node is known as its valence.

In quantum graph theory (QGT) a quantum state describing both the geometry of space and all the matter fields present is built up from combinations of graphs. The theory reached its current form in the work of the Javanese mathematician Kusnanto Sarumpaet, who published a series of six papers from 2035 to 2038 showing that both general relativity and the Standard Model of particle physics could be seen as approximations to QGT.

Sarumpaet's graphs have a fascinating lineage, dating back to Michael Faraday's notion of 'lines of force' running between electric charges, and William Thomson's theory of atoms as knotted 'vortex tubes'. More recent ancestors are Roger Penrose's spin networks, trivalent graphs with each edge labelled by a half-integer, corresponding to a possible value of the spin of a quantum particle. Penrose invented these networks in the early 1970s, and showed that the set of all directions in space could be generated from simple, combinatorial principles by imagining an exchange of spin between two parts of a large network.

Generalizations of spin networks later appeared in certain kinds of quantum field theory. Just as a wavefunction assigns an amplitude to every possible position of a particle, a spin network embedded in a region of space can be used to assign an amplitude to every possible configuration of a field. The quantum states defined in this way consist of lines of flux running along the edges of the network.

In the 1990s, Lee Smolin and Carlo Rovelli discovered an analogous result in quantum gravity, where spin-network states have a simple geometric interpretation: the area of any surface depends entirely on the edges of

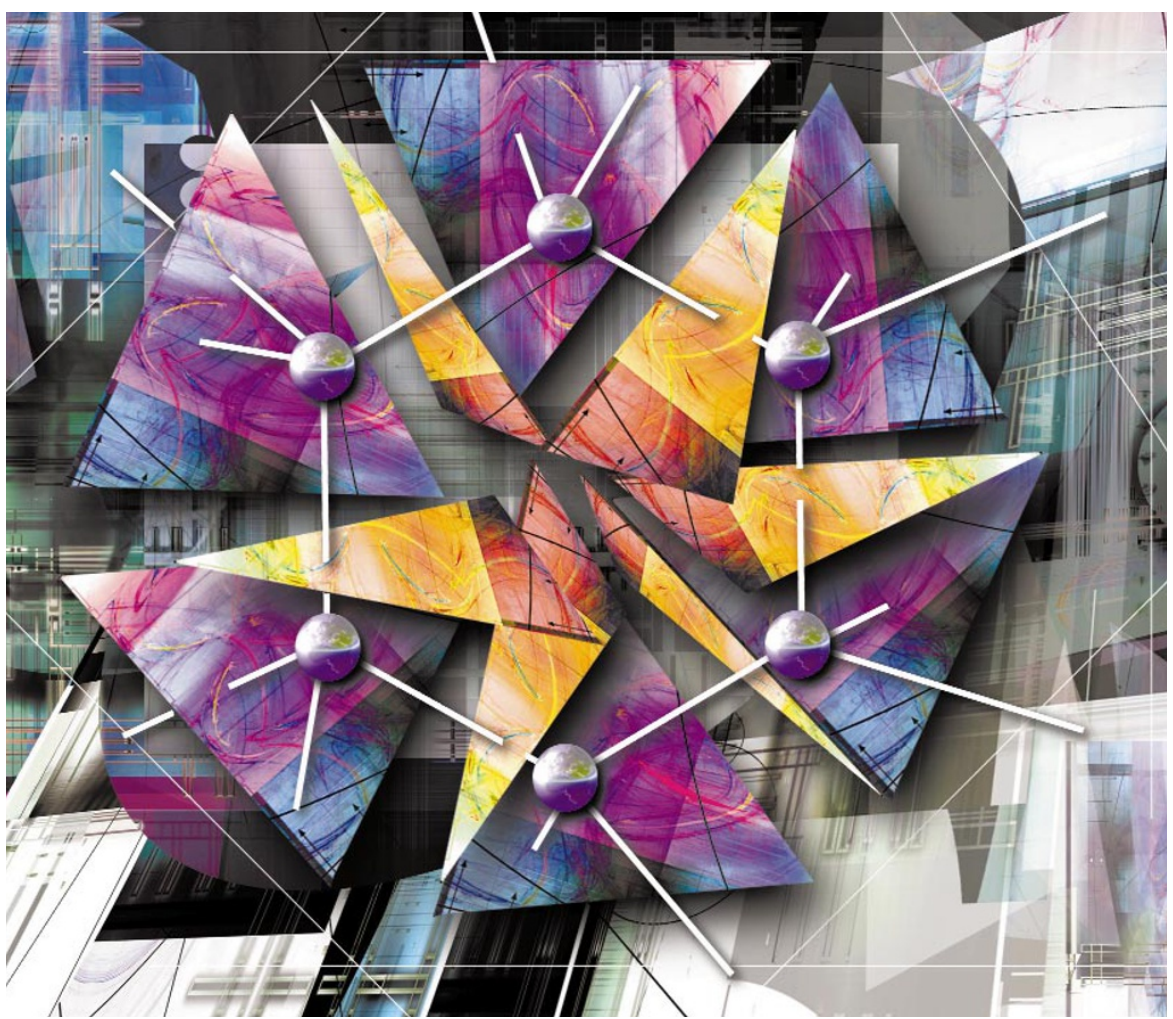

Join the dots: each edge of a quantum graph carries one quantum of area.

the network that intersect it. These edges can be thought of as quantized 'flux lines of area', and in quantum gravity area and other geometric measurements they take on a discrete spectrum of possible values. It then makes sense to quantize the topology as well, with the nodes and edges of the network replacing the usual idea of space as a continuum of points.

In the first decades of the new millennium, John Baez, Fotini Markopoulou, JoséAntonio Zapata and others did groundbreaking work on the possible dynamical laws for spin networks, assigning quantum amplitudes to the process of one network evolving into another. In the 2030s, Sarumpaet began to synthesize these results into a new model, based on graphs of arbitrary valence with unlabelled edges.

The geometry of three-dimensional space arises from tetravalent graphs (pictured), with the four edges emerging from each node giving area to the faces of a 'quantum tetrahedron'. Allowing graphs of higher valence runs the risk of producing an explosion of unwanted dimensions, but Sarumpaet found a simple dynamical law which always leads to the average valence stabilizing at four. However, trivalent and pentavalent nodes - which have come to be known as 'dopant' nodes, in analogy with the impurities added to semiconductors - can persist under the Sarumpaet rules if they are arranged in special patterns: closed, possibly knotted chains of alternating valence. These loops of dopant nodes, classified by their symmetries and mutual interactions, match up perfectly with the particles of the Standard Model.

Since the area associated with the edges of a quantum graph is of the order of a few square Planck lengths, some $10^{-50}$ times the surface area of a hydrogen atom, it was once feared that QGT would remain untestable for centuries. However, in 2043 computer simulations identified a new class of 'polymer states': long, open chains of dopant nodes that were predicted to have energies and half-lives within the grasp of current technology to create and detect.

A search for polymer states that commenced at the Orbital Accelerator Facility in 2049 has now yielded its first success (see $J$. Quant. Graphs 15, 7895-7899; 2050). If the result can be repeated, Sarumpaet's graphs will shift from being merely the most elegant description of the Universe, to being the most likely one.

Greg Egan is a science fiction writer. His latest book is Teranesia (Gollancz). 